\title{
Timing noise as a source of discrepancy between timing and VLBI pulsar positions
}

\author{
A. E. Rodin, Yu. P. Ilyasov, V. V. Oreshko, \\ PRAO ASC LPI, Pushchino, Moscow region, 142292, Russia
}

\author{
M. Sekido
}

KSRC CRL, 893-1, Hirai, Kashima, Ibaraki, 314, Japan

\begin{abstract}
Pulsar positions obtained by VLBI and timing are compared. The difference between these positions is proven to be mainly due to timing noise. A new method which significantly reduces the difference have been developed.
\end{abstract}

A comparison of pulsar VLBI and timing positions is a straightforward way to link the extragalactic and the dynamical coordinate systems. Pulsar coordinates obtained by VLBI and timing differ by an amount which depends on the value of post-fit residuals and strength of timing noise. The timing noise is expressed as long-term variations in TOA (time of arrivals) residuals. Such variations can be described in terms of linear combination of the orthogonal polynomials or the Fourier series. However this can be done only after usual reduction of TOA by the timing analysis software (Tempo (Taylor \& Weisberg 1989), TIMAPR (Doroshenko \& Kopeikin 1990)) because the software does not have possibility to fit long term TOA variations by any kind of orthogonal functions. It means that an inadequate model for parameter fitting is used and in turn results to the pulsar parameters (coordinates $\alpha, \delta$, proper motion $\mu_{\alpha}, \mu_{\delta}$ ) may become biased after ordinal estimation.

Timing observations of a few pulsars were processed in the following manner (Rodin 2000): TOA are processed by TIMAPR software for estimation $\alpha, \delta, \nu$, $\dot{\nu}\left(\mu_{\alpha}, \mu_{\delta}\right.$ were not estimated now because of large timing noise); the residuals obtained were used for the consequent Fourier series fitting; the annual Fourier term yields a correction of pulsar timing coordinates.

In this manner post-fit residuals of a few pulsars were processed. In the Table 1 corrections of its coordinates obtained by method described above (column 2,3 ) and difference between the VLBI (VLA) and timing coordinates (Downs \& Reichly 1983; Fomalont et al. 1992; Ilyasov, Potapov \& Rodin 1999) (column $4,5)$ are presented.

The least square analysis of the data from Table 1 gives the following dependencies of the shift "VLBI - timing" in right ascension $\Delta \alpha_{(V-T)}$ upon the correction made in this paper (column 2 versus 4) $\Delta \alpha_{(V-T)} \cos \delta=$ $0.90( \pm 0.65) \Delta \alpha \cos \delta-0.02( \pm 0.11)$, and in declination (column 3 versus 5 ) $\Delta \delta_{(V-T)}=0.85( \pm 0.18) \Delta \delta+0.10( \pm 0.04)$.

It can be seen there is a strong correlation between offset VLBI - timing and coordinate correction obtained in this paper. On this basis one can conclude 
Table 1. Correction of the pulsar timing coordinates made on the basis of the Fourier analysis of the post-fit residuals and their comparison with difference between the VLBI and timing positions. Coefficient of correlation (column 2 vs. 4) $\rho_{\alpha}=0.61$, confidence $93 \%$. Coefficient of correlation (column 3 vs. 5) $\rho_{\delta}=0.70$, confidence $98 \%$.

\begin{tabular}{|c|cc|cc|}
\hline \multirow{3}{*}{ PSR name } & Coordinate & corrections & \multicolumn{1}{|c|}{ VLBI } & timing \\
\cline { 2 - 5 } & $\begin{array}{c}\Delta \alpha \cos \delta, \\
\operatorname{arcsec}\end{array}$ & $\begin{array}{c}\Delta \delta, \\
\operatorname{arcsec}\end{array}$ & $\begin{array}{c}\Delta \alpha \cos \delta, \\
\operatorname{arcsec}\end{array}$ & $\begin{array}{c}\Delta \delta, \\
\operatorname{arcsec}\end{array}$ \\
\hline 1 & 2 & 3 & 4 & 5 \\
\hline $0329+54$ & $-0.085 \pm 0.006$ & $0.252 \pm 0.026$ & $-0.08 \pm 0.22$ & $0.45 \pm 0.22$ \\
$0834+06$ & $-0.091 \pm 0.049$ & $-0.18 \pm 0.10$ & $0.004 \pm 0.003$ & $-0.1 \pm 0.1$ \\
$0950+08$ & $-0.342 \pm 0.039$ & $-0.30 \pm 0.15$ & $-0.12 \pm 0.15$ & $-0.09 \pm 0.19$ \\
$1133+16$ & $0.025 \pm 0.024$ & $-0.069 \pm 0.033$ & $0.23 \pm 0.14$ & $0.03 \pm 0.22$ \\
$1237+25$ & $-0.026 \pm 0.029$ & $-0.039 \pm 0.025$ & $0.21 \pm 0.13$ & $0.04 \pm 0.16$ \\
$1919+21$ & $0.024 \pm 0.029$ & $-0.057 \pm 0.070$ & $0.008 \pm 0.003$ & $0.2 \pm 0.1$ \\
$1929+10$ & $0.025 \pm 0.003$ & $-0.30 \pm 0.15$ & $-0.12 \pm 0.28$ & $-0.25 \pm 0.27$ \\
$2016+28$ & $-0.294 \pm 0.014$ & $-0.332 \pm 0.041$ & $-0.51 \pm 0.15$ & $-0.03 \pm 0.20$ \\
$2111+46$ & $-0.028 \pm 0.084$ & $0.24 \pm 0.24$ & $-0.40 \pm 0.43$ & $0.23 \pm 0.32$ \\
\hline \hline
\end{tabular}

that inconsistency of VLBI and TOA coordinates is mostly caused by a timing noise of pulsars. After precise fitting of TOA residuals by Fourier series including the term of period 1 year it is possible to make timing position more close to VLBI one. It is believed that pulsar VLBI position reflects the actual positions of the pulsar on the sky and should be used when identifying with objects in other wavelength range.

The timing software used now to process TOA data works well in presence of white noise only meanwhile the most majority of pulsars shows activity not described as pure white noise. For obtaining more reliable pulsar positions from timing data it is necessary to make a modification of the available timing software. The modifications must take into account the non-whiteness of noise presented in TOA's and have possibility to perform a Fourier analysis on the basis of which a correction of pulsar coordinates can be made.

Acknowledgments. Authors express their gratitudes for support the study from RFBR (grant No. 97-02-16446, 98-02-16537) and Ministry of Science RF.

\section{References}

Doroshenko, O. V., \& Kopeikin, S. M. 1990, Sov. Astr., 34, 496.

Downs, G. S., Reichly, P. E. 1983, ApJ, 53, 169.

Fomalont, E. B., Goss, W. G., Lyne, A. G., R. N. Manchester, R. N., \& Justtanont, K. 1992, MNRAS, 258, 497.

Ilyasov, Yu. P., Potapov, V. A., \& Rodin, A. E. 1999, This proceedings.

Rodin, A. E. 2000, PhD thesis, P. N. Lebedev Physical Institute.

Taylor, J. H., \& Weisberg, J. M. 1989, ApJ, 345, 434. 